\title{
Energy balance and cascade in MHD turbulence in the solar corona
}

\author{
Francesco Malara, Giuseppina Nigro and Pierluigi Veltri \\ Dipartimento di Fisica, Università della Calabria, \\ via P. Bucci, I-87036, Rende (CS), Italy \\ email: malara@fis.unical.it, nigro@fis.unical.it, veltri@fis.unical.it
}

\begin{abstract}
The dynamics of fluctuations in a closed coronal structure is regulated both by resonance with motions at bases that stores energy in the structure in form of discrete eigenmodes, and by nonlinear couplings that move this energy along the spectrum to smaller scales. The energy balance is evaluated both analytically and, numerically, using an hybrid shell model. The input energy flux is independent of nonlinear effects and is determined by slow (DC) perturbations. Coherent eigenmode couplings determine the nonlinear energy flux and, consequently, the level of fluctuations at large scales. The estimated velocity fluctuation level is in agreement with measures of nonthermal velocity in corona. The resulting turbulence spectrum contains both a pre-inertial range where coherent interactions dominate, and a standard inertial range where the turbulence behaves as in an unbounded system.
\end{abstract}

Keywords. Sun: corona, (magnetohydrodynamics:) MHD, turbulence, waves

\section{Introduction}

Within the theoretical modelling of coronal heating, there are two important questions that are currently debated: first, in which form and at which time scale the energy of photospheric motions reaches the corona; second, which are the mechanisms that carry this energy to the very small dissipative scales. Concerning the former point, models of coronal heating have been traditionally classified in two groups: 1) DC models consider slow photospheric motions at time scales $t_{p h} \gg T_{A}, T_{A}$ being the Alfvén crossing time. The coronal structures continuously re-arrange through a sequence of quasi-equilibrium states, while the magnetic free energy increases in time; energy enters the corona mainly as magnetic energy. 2) AC heating models consider perturbations at $t_{p h} \lesssim T_{A}$; kinetic energy is comparable with magnetic energy. Due to the finite length of closed coronal structures, exciting such perturbations is similar to put in resonance an elastic string of given length by an external driver (Ionson 1982). Typically, the Alfvén crossing time $T_{A} \sim 10-15 \mathrm{~s}$, while the energy of photospheric motions is concentrated around $t_{p h} \sim 300$ $\mathrm{s}$; this suggests that photospheric motions are expected to energize the corona mainly by DC perturbations. However, the distinction between AC and DC mechanisms is quite artificial (Milano et al. 1997) because, in the context of resonant systems a DC mechanism can be simply considered as corresponding to a resonance at zero frequency.

The second question concerns mechanisms that move energy towards small enough scales (probably of the order of the proton Larmor radius), where it can be efficiently dissipated. Linear mechanisms related to the interaction between perturbations and an inhomogeneous background are: (i) resonant absorption (e.g., Davila 1987), in which the perturbation energy concentrates around magnetic fieldlines where the wave frequency matches the local Alfvén frequency; (ii) phase-mixing (e.g., Heyvaerts \& Priest 1983) where differences in the local phase velocity bend wavefronts progressively decreasing the transverse wavelength; (iii) 3D fast dissipation (Petkaki et al. 1998), where exponential 
separation of nearby magnetic lines builds up small scales faster than in ordinary phasemixing. In contrast, turbulence models assume a uniform background and study how nonlinear interactions build up an energy cascade from large to small dissipative scales (Einaudi et al. 1996, Dmitruk \& Gomez 1997, Nigro et al. 2004). The injection range of the turbulence corresponds to the length of the photospheric velocity pattern: $\sim\left(1.5 \times 10^{4}\right.$ $\left.1.5 \times 10^{3}\right) \mathrm{km}$, the dissipative length is of the order of the proton Larmor radius $(\sim(10-1)$ $\mathrm{m})$; the inertial range of the turbulence, formed by several decades, where nonlinear interactions play the major role, is located in between.

A model of coronal heating should include both the aspect of energization of the corona and that of the internal dynamics which leads to dissipation. Milano et al. (1997) considered a model of a coronal loop with a spectrum of driving frequencies ranging from low (DC) to higher (AC) values. Resonances play a key role allowing for the fluctuating energy to enter the loop. The resulting turbulence, described by an EDQNM technique, moves energy to microscales and it has the net effect of an enhanced dissipation on macroscales.

Direct numerical simulations give a detailed representation of spatial structures, but cannot adequately describe the wide range of spatial scales of the coronal turbulence. This is more easily represented in "reduced" models, like shell models (Giuliani \& Carbone 1998, Boffetta et al. 1999), in which the dynamics of turbulence is represented in a simplified Fourier space. A drawback of shell models is that phenomena related to wave propagation, like resonance, cannot be described due to the lack of spatial information. A compromise is represented by the hybrid shell model (Nigro et al. 2004, Buchlin \& Velli 2007), based on the reduced MHD (RMHD) equations, that includes both nonlinear effects using a shell technique, and linear propagation along the magnetic field. This gives an adequate description of a wide turbulent range as well as of the resonance phenomenon. In the present paper we focus on the energy input due to motions at the loop basis at large scales and on the energy flux towards small scales due to nonlinear effects, deriving, by an analytical treatment, some properties of fluctuations and of nonlinear interactions at large scales. The results are compared with those derived from the hybrid shell model (Nigro et al. 2004).

\section{RMHD and energy balance equations}

A coronal magnetic structure is characterized by a strong longitudinal magnetic field, by a large aspect ratio $R=L / L_{\perp}$ ( $L$ and $L_{\perp}$ being the longitudinal and the transverse lengths, respectively), and by a low value of the plasma $\beta\left(\sim 10^{-2}\right)$. In these conditions the large scale dynamics can be described by the RMHD equations (Strauss 1976), that are written in the following form, after a Fourier expansion in the transverse directions:

$$
\begin{gathered}
\rho_{0} \frac{\partial v_{n}\left(\mathbf{k}_{\perp}\right)}{\partial t}-\frac{B_{0}}{4 \pi} \frac{\partial B_{n}\left(\mathbf{k}_{\perp}\right)}{\partial z} \\
=-i k_{\perp n} P\left(\mathbf{k}_{\perp}\right)+\sum_{\mathbf{p}_{\perp}, \mathbf{q}_{\perp}} i q_{\perp j}\left[\frac{1}{4 \pi} B_{j}\left(\mathbf{p}_{\perp}\right) B_{n}\left(\mathbf{q}_{\perp}\right)-\rho_{0} v_{j}\left(\mathbf{p}_{\perp}\right) v_{n}\left(\mathbf{q}_{\perp}\right)\right] \delta_{\mathbf{p}_{\perp}+\mathbf{q}_{\perp}, \mathbf{k}_{\perp}} \\
\frac{\partial B_{n}\left(\mathbf{k}_{\perp}\right)}{\partial t}-B_{0} \frac{\partial v_{n}\left(\mathbf{k}_{\perp}\right)}{\partial z}=\sum_{\mathbf{p}_{\perp}, \mathbf{q}_{\perp}} i q_{\perp j}\left[B_{j}\left(\mathbf{p}_{\perp}\right) v_{n}\left(\mathbf{q}_{\perp}\right)-v_{j}\left(\mathbf{p}_{\perp}\right) B_{n}\left(\mathbf{q}_{\perp}\right)\right] \delta_{\mathbf{p}_{\perp}+\mathbf{q}_{\perp}, \mathbf{k}_{\perp}}
\end{gathered}
$$

where $\mathbf{v}$ and $\mathbf{B}$ are the transverse velocity and magnetic field, $P$ is the total (fluid + magnetic) pressure, $z$ is the longitudinal coordinate. These equations indicate that the dynamics of perturbations is determined by two mechanisms: (i) linear propagation along 
$\pm \mathbf{B}_{0}$ at the Alfvén velocity $c_{A 0}=B_{0} /\left(4 \pi \rho_{0}\right)^{1 / 2}$ (LHS terms); (ii) nonlinear couplings between Fourier modes at different transverse wavevectors (RHS terms). The kinetic and magnetic energy at a given transverse wavevector $\mathbf{k}_{\perp}$ are:

$$
E_{k i n}\left(\mathbf{k}_{\perp}, t\right)=\frac{1}{2} \rho_{0} L_{\perp}^{2} \int_{0}^{L} d z\left|v_{n}\left(\mathbf{k}_{\perp}, z, t\right)\right|^{2} ; \quad E_{\text {mag }}\left(\mathbf{k}_{\perp}, t\right)=\frac{L_{\perp}^{2}}{8 \pi} \int_{0}^{L} d z\left|B_{n}\left(\mathbf{k}_{\perp}, z, t\right)\right|^{2}
$$

whose time evolution satisfies the energy balance equations:

$$
\frac{d E_{k i n}\left(\mathbf{k}_{\perp}\right)}{d t}=L_{\perp}^{2}\left[\Phi_{k i n}^{i n}\left(\mathbf{k}_{\perp}\right)+\Phi_{k i n}^{n l}\left(\mathbf{k}_{\perp}\right)\right] ; \quad \frac{d E_{m a g}\left(\mathbf{k}_{\perp}\right)}{d t}=L_{\perp}^{2}\left[\Phi_{m a g}^{i n}\left(\mathbf{k}_{\perp}\right)+\Phi_{m a g}^{n l}\left(\mathbf{k}_{\perp}\right)\right]
$$

where $\Phi_{k i n}^{n l}$ and $\Phi_{m a g}^{n l}$ are the spectral energy fluxes due to nonlinear effects, defined by

$$
\begin{aligned}
\Phi_{k i n}^{n l}\left(\mathbf{k}_{\perp}\right)= & \frac{1}{2} \int_{0}^{L} d z \sum_{\mathbf{p}_{\perp}, \mathbf{q}_{\perp}} i q_{\perp j}\left[\rho_{0}\left[v_{n}\left(\mathbf{k}_{\perp}\right) v_{j}^{*}\left(\mathbf{p}_{\perp}\right) v_{n}^{*}\left(\mathbf{q}_{\perp}\right)-v_{n}^{*}\left(\mathbf{k}_{\perp}\right) v_{j}\left(\mathbf{p}_{\perp}\right) v_{n}\left(\mathbf{q}_{\perp}\right)\right]\right. \\
& \left.+\frac{1}{4 \pi}\left[v_{n}^{*}\left(\mathbf{k}_{\perp}\right) B_{j}\left(\mathbf{p}_{\perp}\right) B_{n}\left(\mathbf{q}_{\perp}\right)-v_{n}\left(\mathbf{k}_{\perp}\right) B_{j}^{*}\left(\mathbf{p}_{\perp}\right) B_{n}^{*}\left(\mathbf{q}_{\perp}\right)\right]\right] \delta_{\mathbf{p}_{\perp}+\mathbf{q}_{\perp}, \mathbf{k}_{\perp}}
\end{aligned}
$$

and

$$
\begin{aligned}
\Phi_{m a g}^{n l}\left(\mathbf{k}_{\perp}\right)= & \frac{1}{8 \pi} \int_{0}^{L} d z \sum_{\mathbf{p}_{\perp}, \mathbf{q}_{\perp}} i q_{\perp j}\left[B_{n}\left(\mathbf{k}_{\perp}\right) v_{j}^{*}\left(\mathbf{p}_{\perp}\right) B_{n}^{*}\left(\mathbf{q}_{\perp}\right)-B_{n}^{*}\left(\mathbf{k}_{\perp}\right) v_{j}\left(\mathbf{p}_{\perp}\right) B_{n}\left(\mathbf{q}_{\perp}\right)\right. \\
& \left.+B_{n}^{*}\left(\mathbf{k}_{\perp}\right) B_{j}\left(\mathbf{p}_{\perp}\right) v_{n}\left(\mathbf{q}_{\perp}\right)-B_{n}\left(\mathbf{k}_{\perp}\right) B_{j}^{*}\left(\mathbf{p}_{\perp}\right) v_{n}^{*}\left(\mathbf{q}_{\perp}\right)\right] \delta_{\mathbf{p}_{\perp}+\mathbf{q}_{\perp}, \mathbf{k}_{\perp}}
\end{aligned}
$$

while the input energy flux through the loop bases is

$$
\Phi_{\text {tot }}^{i n}\left(\mathbf{k}_{\perp}\right)=\Phi_{\text {kin }}^{i n}\left(\mathbf{k}_{\perp}\right)+\Phi_{m a g}^{i n}\left(\mathbf{k}_{\perp}\right)=\frac{B_{0}}{4 \pi}\left[\Re\left[v_{n}\left(\mathbf{k}_{\perp}\right) B_{n}^{*}\left(\mathbf{k}_{\perp}\right)\right]_{z=0}^{z=L}\right]
$$

Since only one variable $(v$ or $B$ ) can be specified at each boundary, the input flux is determined both by the boundary conditions (representing the external forcing), and by the internal dynamics of the system.

\section{Linear dissipative model}

We consider the velocity and the magnetic field at a given transverse wavevector $\mathbf{k}_{\perp}$ within the energy injection range $k_{\perp} \sim 2 \pi / l_{\perp}^{i n}$. Nonlinear couplings represented by nonlinear terms in equations (2.1), (2.2) put away energy from this mode towards smaller transverse scales. We assume that in the injection range these nonlinear terms are small with respect to the linear ones (weak nonlinearity). Thus, a simplified model can be derived (Milano et al. 1997), in which nonlinear terms in equations (2.1) and (2.2) are replaced by fictitious linear dissipative terms:

$$
\frac{\partial v_{\perp}}{\partial t}=\frac{B_{0}}{4 \pi \rho_{0}} \frac{\partial B_{\perp}}{\partial z}-\nu k_{\perp}^{2} v_{\perp} ; \quad \frac{\partial B_{\perp}}{\partial t}=B_{0} \frac{\partial v_{\perp}}{\partial z}-\lambda k_{\perp}^{2} B_{\perp}
$$

$v_{\perp}(z, t)$ and $B_{\perp}(z, t)$ are real quantities representing the real or the imaginary part of perturbations, while the fictive dissipative coefficients $\nu$ and $\lambda$ are small quantities and represent free parameters. Boundary conditions give the velocity perturbation at the loop ends:

$$
v_{\perp}(z=0, t)=u(t) ; \quad v_{\perp}(z=L, t)=0
$$

where $u(t)$ is a random gaussian-distributed signal with a given auto-correlation time $T_{c}$. 
We assume the following ordering among characteristic times

$$
T_{A} \ll T_{c} \ll T_{d}, T_{d 0}
$$

where $T_{A}=L / c_{A 0}$ is the Alfvén crossing time and $T_{d}=1 / \omega_{d}=2 /(\nu+\lambda) k_{\perp}^{2}, T_{d 0}=$ $1 / \omega_{d}^{(0)}=1 /\left(\lambda k_{\perp}^{2}\right.$ are the dissipative times giving a measure of the nonlinear time $\left.T_{n l}\right)$.

The results derived from the linear model can be summarized as follows. In the weak nonlinearity limit perturbations are a superposition of eigenmodes, each at frequency $\omega_{n}=\left(c_{A 0} \pi / L\right) n$, representing Alfvén standing waves; at $\omega_{n}=0$ the velocity is a linear function of $z$ while magnetic field is constant. Eigenmodes have a well-defined parity in space and time and their amplitude is determined by dissipative coefficients, i.e., by nonlinear effects (Nigro et al. 2008).

The input energy flux $\left\langle\Phi_{\text {tot }}^{i n}\right\rangle_{t}$ is a convolution in the frequency space between the spectrum $G(\omega)=2 u_{0} T_{c} /\left(1+\omega^{2} T_{c}^{2}\right)$ of velocity at the loop base ( $u_{0}$ being the velocity amplitude) and a function $H_{u B}(\omega)$ describing the response of the loop at each frequency $\omega_{n}$. The main contribution to $\left\langle\Phi_{\text {tot }}^{i n}\right\rangle_{t}$ is due to the resonance at $\omega_{n}=0$ (DC motions), which gives (Nigro et al. 2008):

$$
\left\langle\Phi_{\text {tot }}^{i n}\right\rangle_{t} \simeq-\frac{B_{0}^{2}}{4 \pi} \frac{T_{c}}{L} u_{0}^{2}
$$

This expression is independent of $T_{d}$ and $T_{d 0}$. Thus, the input flux does not depend on nonlinear effects but only on the loop resonance. The scaling law (3.4) is the same as in "stochastic buildup models" (Sturrock \& Uchida 1981), where random photospheric motions increase the magnetic energy by twisting flux tubes. Assuming $B_{0}=100 \mathrm{G}$, $L=3 \times 10^{9} \mathrm{~cm}, T_{c}=300 \mathrm{~s}$ and $u_{0}=10^{5} \mathrm{~cm} \mathrm{~s}^{-1}$, we get $\left\langle\Phi_{\text {tot }}^{\text {in }}\right\rangle_{t}=8 \times 10^{5} \mathrm{erg} \mathrm{cm}^{-2}$ $\mathrm{s}^{-1}$, which is of the order of the energy flux required to sustain the quiet-Sun corona (Withbroe 1988).

The main contribution to fluctuating kinetic energy comes from the $n=1$ eigenmode (AC), which gives an estimation for the velocity fluctuation:

$$
\delta v \simeq u_{0}\left(\frac{T_{d}}{6 T_{c}}\right)^{1 / 2}
$$

We note that $\delta v \gg u_{0}$, in accordance with measures of nonthermal velocities in corona much larger than photospheric velocities. Magnetic fluctuations are mainly due to the $n=0$ eigenmode (DC), giving an estimation for the magnetic field fluctuation:

$$
\delta B \simeq \frac{B_{0}}{c_{A}} u_{0}\left(\frac{T_{c} T_{d 0}}{2 T_{A}^{2}}\right)^{1 / 2}
$$

Since $\delta B / B_{0} \gg \delta v / c_{A}$ the turbulence injection range is magnetically dominated. Note that both $\delta v$ and $\delta B$ depend on dissipative coefficients, i.e., are determined by the amplitude of nonlinear effects.

\section{Nonlinear effects: spectral energy fluxes}

In a statistically stationary situation, the input energy flux must be balanced by the spectral flux moving energy from the whole injection range to smaller scales along the spectrum. The spectral flux can be estimated using the expressions (2.5), (2.6), as well as the form of eigenmodes derived from the linear analysis. Nonlinear interactions take place among triads of wavevectors $\left(\mathbf{k}_{\perp}, \mathbf{p}_{\perp}, \mathbf{q}_{\perp}\right)$. The perturbation at a given wavevector is a superposition of infinite eigenmodes. However, due to the symmetry properties of 
eigenmode profiles, nonlinear interactions are dominated by coherence effects. This fact has some consequences that make this situation peculiar with respect to a "standard" MHD turbulence (Nigro et al. 2008): (i) in each interacting triad, two AC eigenmodes $(n \neq 0)$ interact with the velocity perturbation of a DC $(n=0)$ eigenmode; (ii) only eigenmodes with the same parallel wavelength $($ at $n \neq 0)$ can efficiently interact; in the other cases the energy flux is vanishing; (iii) only interactions where two wavevectors are in the injection range and the third in the inertial range contribute to the energy flux; (iv) the kinetic and magnetic spectral energy fluxes are equal; (v) the above properties makes the spectral energy flux much smaller than in a standard MHD turbulence; this justifies the hypothesis of weak nonlinearity (equation (3.3)).

As a consequence, the turbulence spectrum is characterized by the presence of a preinertial range at scales just smaller than those of the injection range. In the pre-inertial range nonlinear interactions are strongly influenced by coherence effects, due to the presence of linear eigenmodes. In particular, in the pre-inertial range magnetic energy dominates kinetic energy, as is the injection range. With increasing $k_{\perp}$ the nonlinear time $T_{n l}$ decreases, while $T_{A}$ keeps unchanged; this implies that at smaller scales resonance lines becomes wider and wider. When $T_{n l}$ becomes $\lesssim T_{A}$ nonlinear effects dominate linear ones: resonant eigenmodes disappear and the turbulence is determined by incoherent mode interactions; this corresponds to a standard inertial range, where $E_{k i n} \sim E_{\text {mag }}$ and the two spectra both follow a Kolmogorov law. The transition between pre-inertial and inertial range is characterized by $T_{n l} \sim T_{A}$ giving the condition

$$
k_{\perp}^{i n c} \delta v\left(k_{\perp}^{i n c}\right) \sim \frac{L}{c_{A 0}}
$$

where $k_{\perp}^{i n c}$ is the lower limit of the pre-inertial range. An estimation of the spectral energy flux from the injection range to smaller scales is (Nigro et al. 2008):

$$
\left\langle\Phi_{t o t}^{n l}\right\rangle_{t} \simeq \frac{2 \pi}{\sqrt{3}} \frac{L}{l_{\perp}^{i n}} \rho_{0} u_{0} \delta v^{2}\left(l_{\perp}^{i n}\right)
$$

$\rho_{0}$ being the background density. Energy conservation requires $\left\langle\Phi_{\text {tot }}^{i n}\right\rangle_{t}+\left\langle\Phi_{\text {tot }}^{n l}\right\rangle_{t}=0$. Then, from the expressions (3.4) and (4.2) we obtain a scaling law for the velocity fluctuation in the injection range:

$$
\delta v\left(l_{\perp}^{i n}\right) \simeq\left(\frac{\sqrt{3}}{2 \pi} \frac{l_{\perp}^{i n}}{L} \frac{T_{c}}{T_{A}} c_{A 0} u_{0}\right)^{1 / 2}
$$

Using the values $l_{\perp}^{i n}=3 \times 10^{8} \mathrm{~cm}, L=3 \times 10^{9} \mathrm{~cm}, T_{c}=300 \mathrm{~s}, T_{A}=15 \mathrm{~s}, c_{A 0}=2 \times 10^{8}$ $\mathrm{cm} \mathrm{s}^{-1}, u_{0}=10^{5} \mathrm{~cm} \mathrm{~s}^{-1}$ we obtain $\delta v\left(l_{\perp}^{i n}\right)=3 \times 10^{6} \mathrm{~cm} \mathrm{~s}^{-1}$, which is consistent with typical nonthermal velocities measured in the corona (e.g., Warren et al. 1997). Since the spectral flux is independent of the amplitude of DC magnetic perturbation, this method does not allow us to obtain a scaling law for $\delta B$.

\section{A numerical approach: the hybrid shell model}

The hybrid shell model (Nigro et al. 2004, Buchlin \& Velli 2007) is based on RMHD equations (2.1), (2.2); propagation terms in the parallel $(z)$ direction are explicitly included, while the perpendicular spectral space is divided into concentric shell of exponentially increasing width. At each shell a value $k_{\perp n}=k_{0} 2^{n}\left(k_{0}=2 \pi / l_{\perp}^{i n}, n=\right.$ $\left.0,1, \ldots, n_{\max }\right)$ of the wavevector is assigned, while velocity and magnetic fluctuations (normalized to $c_{A 0}$ and to $B_{0}$, respectively) are represented by complex scalar quantities $v_{\perp n}(z, t), b_{\perp n}(z, t)$. Nonlinear couplings are represented by quadratic nonlinear terms 

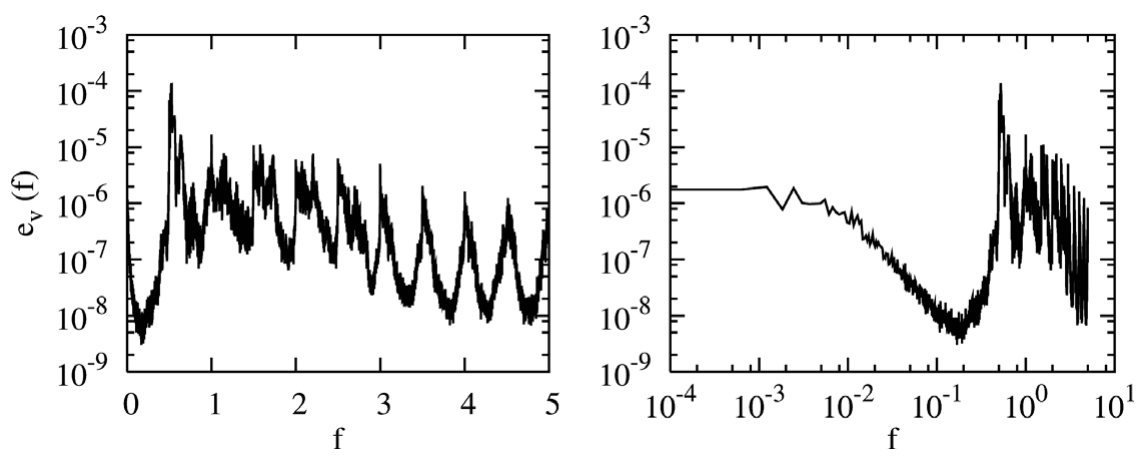

Figure 1. Velocity spectrum $e_{v}$ as a function of the normalized frequency $f=\omega L / 2 \pi a_{A 0}$ in semilogarithmic scale (left) and in logarithmic scale (right).

whose coefficients are chosen in order to have conservation of $2 \mathrm{D}$ quadratic invariants. The equations of the hybrid shell model are (Nigro et al. 2004):

$$
\begin{aligned}
\left(\frac{\partial}{\partial t} \mp \frac{\partial}{\partial z}\right) Z_{n}^{ \pm}=i k_{\perp n}\left(\frac{13}{24} Z_{n+2}^{ \pm} Z_{n+1}^{\mp}\right. & +\frac{11}{24} Z_{n+2}^{\mp} Z_{n+1}^{ \pm}-\frac{19}{48} Z_{n+1}^{ \pm} Z_{n-1}^{\mp}-\frac{11}{48} Z_{n+1}^{\mp} Z_{n-1}^{ \pm} \\
& \left.+\frac{19}{96} Z_{n-1}^{ \pm} Z_{n-2}^{\mp}+\frac{13}{96} Z_{n-1}^{\mp} Z_{n-2}^{ \pm}\right)+\chi k_{\perp n}^{2} Z_{n}^{ \pm}
\end{aligned}
$$

where $Z_{n}^{ \pm}=v_{\perp n} \pm b_{\perp n}$ are the Elsässer variables, $\chi$ is a dissipative coefficient which determine the dissipative scale, $z$ is normalized to $L$ and $t$ to $L / c_{A 0}$. Boundary conditions are the same as in the analytical treatment (equation 3.2) and are imposed on the first three shells; then, these shells represent the energy injection range of the turbulence.

The hybrid shell model is able to reproduce both nonlinear effects and linear phenomena, like resonance, that are naturally included into the model. Then, results derived from it can be compared with those of the analytical treatment previously described; in particular, the hypothesis of weak nonlinearity and the related dominance of resonant eigenmodes at large scales. Moreover, a wide spectral range can be described with a limited numerical effort; this allows to get a detailed description of the spectra.

The evolution equations (5.1) have been numerically solved, using the following values for the parameters: $L=3 \times 10^{9} \mathrm{~cm}, L_{\perp}=\pi \times 10^{8} \mathrm{~cm}, c_{A 0}=2 \times 10^{8} \mathrm{~cm} \mathrm{~s}^{-1}, \rho_{0}=$ $1.67 \times 10^{-16} \mathrm{~g} \mathrm{~cm}^{-3}, u_{0}=10^{5} \mathrm{~cm} \mathrm{~s}^{-1}, T_{c}=300 \mathrm{~s}, \chi=2 \times 10^{-9}$. This value of $\chi$ gives a dissipative length $l_{d}=1.2 \times 10^{3} \mathrm{~cm}$, which is a factor $\simeq 4 \times 10^{-6}$ smaller than the injection scale. Such a wide range of scales for the turbulence ( 6 decades) is inaccessible to direct MHD numerical simulations.

From the space-time dependence of fluctuations $v_{\perp n}(z, t), b_{\perp n}(z, t)$ in each shell, we calculated the frequency spectra $e_{v}(\omega)$ of velocity and $e_{b}(\omega)$ of magnetic field, integrated in space. We select the shell $n=1$ which is at the middle of the energy injection range:

$$
e_{v}(\omega)=\int_{0}^{1} d z\left|\hat{v}_{\perp 1}(z, \omega)\right|^{2} ; \quad e_{b}(\omega)=\int_{0}^{1} d z\left|\hat{b}_{\perp 1}(z, \omega)\right|^{2}
$$

where $\hat{v}_{\perp 1}$ and $\hat{b}_{\perp 1}$ are the Fourier time transform of velocity and magnetic field fluctuations:

$$
v_{\perp 1}(z, t)=\sum_{\omega} \hat{v}_{\perp 1}(z, \omega) e^{-i \omega t} ; \quad b_{\perp 1}(z, t)=\sum_{\omega} \hat{b}_{\perp 1}(z, \omega) e^{-i \omega t}
$$

The velocity frequency spectrum $e_{v}$ is plotted in Figure 1, as a function of the normalized frequency $f=\omega L / 2 \pi a_{A 0}$. The spectrum displays a sequence of peaks approximately 

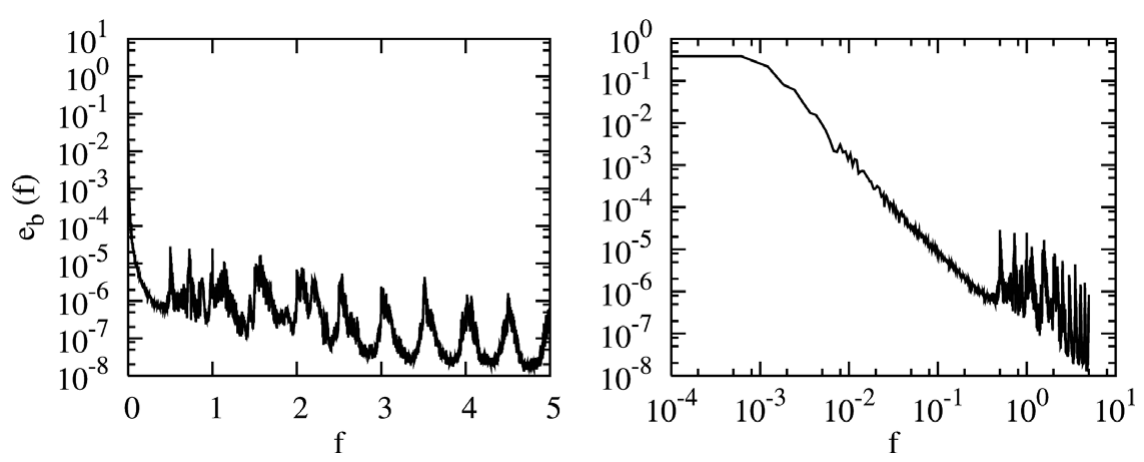

Figure 2. The same as in Figure 1, for the magnetic field spectrum $e_{b}$.

located at semi-integer values of $f$, that corresponds to the resonance frequencies $\omega_{n}$. This clearly show the presence of resonant eigenmodes that dominate large scale fluctuations. The most energetic eigenmode corresponds to the 1st-order resonance, while much less energy is present in the 0th-order resonance. Thus, in accordance with the analytical model, AC perturbations (in particular, the $n=1$ resonance) dominate the velocity fluctuations. The width of the dominant peak is much less than the separation $T_{A}^{-1}$ between peaks; this indicates that at large scales the nonlinear time is much longer than $T_{A}$, thus supporting the assumption of weak nonlinearity made in the analytical treatment.

The magnetic field frequency spectrum $e_{b}$ is plotted in Figure 2, as a function of $f$. Also this spectrum is formed by a sequence of peaks localized at the resonance frequencies (semi-integer $f$ ). In contrast with the velocity spectrum, magnetic fluctuations are dominated by the resonant eigenmode $n=0$, corresponding to slow DC perturbations. Comparing the two spectra we see that, at resonance frequencies $n \neq 0, e_{v} \sim e_{b}$ (except for $n=1$ where $e_{v}$ is slightly larger than $e_{b}$ ), this indicates that resonant AC perturbations are stationary Alfvén waves trapped into the closed magnetic structure. At the resonance $n=0$ we find $e_{b} \gg e_{v}$, as expected. All these features are in accordance with the results of the analytical model.

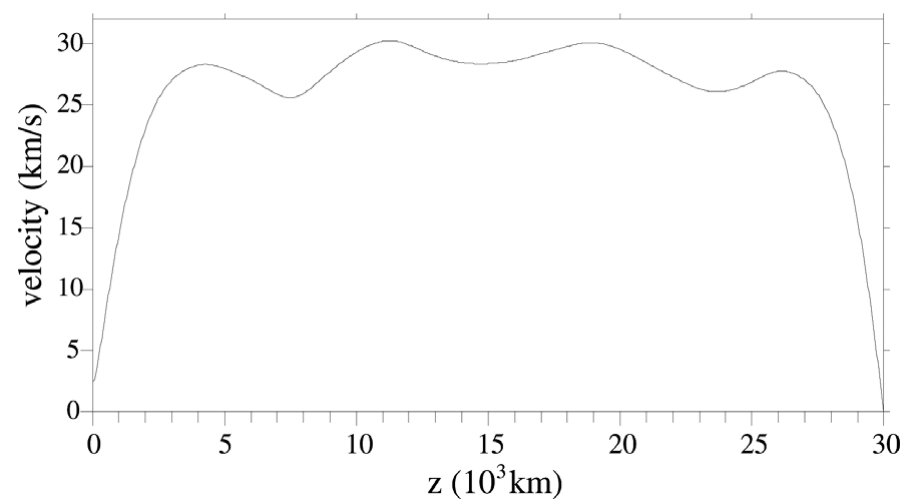

Figure 3. Spatial profile of rms velocity perturbation $\delta v$ as a function of the longitudinal coordinate $z$. 
In Figure 3 we show the spatial profile of the rms velocity perturbation, defined by

$$
\delta v(z)=\left(\int_{0}^{T} \frac{d t}{T} \sum_{n}\left|v_{\perp n}(z, t)\right|^{2}\right)^{1 / 2}
$$

The contribution of the resonant eigenmode profiles at $n \lesssim 4$ can be recognized in Figure 3. The typical value for the velocity perturbation found in the run is $\delta v \simeq 3 \times 10^{6}$ $\mathrm{cm} \mathrm{s}^{-1}$, which is in accordance with the estimation (4.3) derived from the analytical treatment. As expected, this value of $\delta v$ is much larger than at the loop basis, which is of the order of $10^{5} \mathrm{~cm} \mathrm{~s}^{-1}$.

The rms magnetic field perturbation found numerically is $\delta B / B_{0} \simeq 0.2 \gg \delta v / c_{A 0}$. This value of $\delta B$ is mainly due to the $n=0$ resonance; for this reason, such a value cannot be predicted using energy flux conservation, as we did for $\delta v$.

The total energy $E$, the net incoming power $F$ and the dissipated power $W$ are shown in Figure 4 as functions of time. During a transient, which lasts about 15 hours, the energy increases. For subsequent times the energy does not stabilize but displays strong irregular variations. These variations are due both to energy input/output at the lower boundary and to dissipation. The energy input depends on how the forcing at the base couples with perturbations which are present inside the system. This coupling contributes to determine the sign of the incoming power $F$ which continuously changes sign on a short time scale (Figure 4). Excluding the initial transient, the time average of net incoming and dissipated powers are $\langle F\rangle_{t} \sim\langle W\rangle_{t} \simeq 2.6 \times 10^{22} \mathrm{erg} \mathrm{s}^{-1}$. We compare this value with the predictions of the analytical model; from equation (3.4) we derive

$$
\langle F\rangle_{t} \sim \frac{B_{0}^{2}}{4 \pi c_{A 0}} \frac{T_{c}}{T_{A}} \delta u^{2} L_{\perp}^{2}
$$

Using the above values for the parameters, this equation gives $\langle F\rangle_{t} \sim 2 \times 10^{22} \mathrm{erg} \mathrm{s}^{-1}$, which is quite close to the value of the input power in the hybrid shell model. Then, the analytical estimation (3.4) of the input energy flux is in agreement with the results of the numerical model.

Motions at the loop basis inject energy at scales $\sim l_{\perp}^{i n}$ and nonlinear couplings transfer this energy to smaller transverse scales forming a spectrum which extends down to dissipative scales. The average spectra of kinetic and magnetic fluctuations are defined by

$$
\left\langle e_{n}^{(v)}\right\rangle_{t}=\int_{0}^{1} d z \int_{0}^{T} \frac{d t}{T}\left|v_{\perp n}(z, t)\right|^{2} ; \quad\left\langle e_{n}^{(b)}\right\rangle_{t}=\int_{0}^{1} d z \int_{0}^{T} \frac{d t}{T}\left|b_{\perp n}(z, t)\right|^{2}
$$

These perpendicular spectra are shown in Figure 5 . Note that a linear scale in the index $n$ corresponds to a logarithmic scale in $k_{\perp n}$. In the injection range $(0 \leqslant n \leqslant 2)$ magnetic fluctuations dominate velocity fluctuations, but the average slope of $\left\langle e_{n}^{(b)}\right\rangle_{t}$ is much larger than that of $\left\langle e_{n}^{(v)}\right\rangle_{t}$. Thus, for increasing $n$ there is a tendency to an equipartition between $\left\langle e_{n}^{(b)}\right\rangle_{t}$ and $\left\langle e_{n}^{(v)}\right\rangle_{t}$, which is approximately verified in the range $5 \lesssim n \lesssim 10$. Within such a range both the kinetic and the magnetic energy spectra approximately follow a Kolmogorov law, in accordance with what expected from the analytical treatment. The kinetic energy spectrum has a similar slope also in the injection range. In the considered case we have $l_{\perp}^{(n r)} \sim 2^{-5} L_{\perp 0} \simeq 2 \times 10^{7} \mathrm{~cm}$. Then, from equation (4.1) we find $\delta v\left(l_{\perp}^{(n r)}\right) \sim$ $1.3 \times 10^{6} \mathrm{~cm} \mathrm{~s}^{-1}$, which is compatible with the results of the numerical model. 


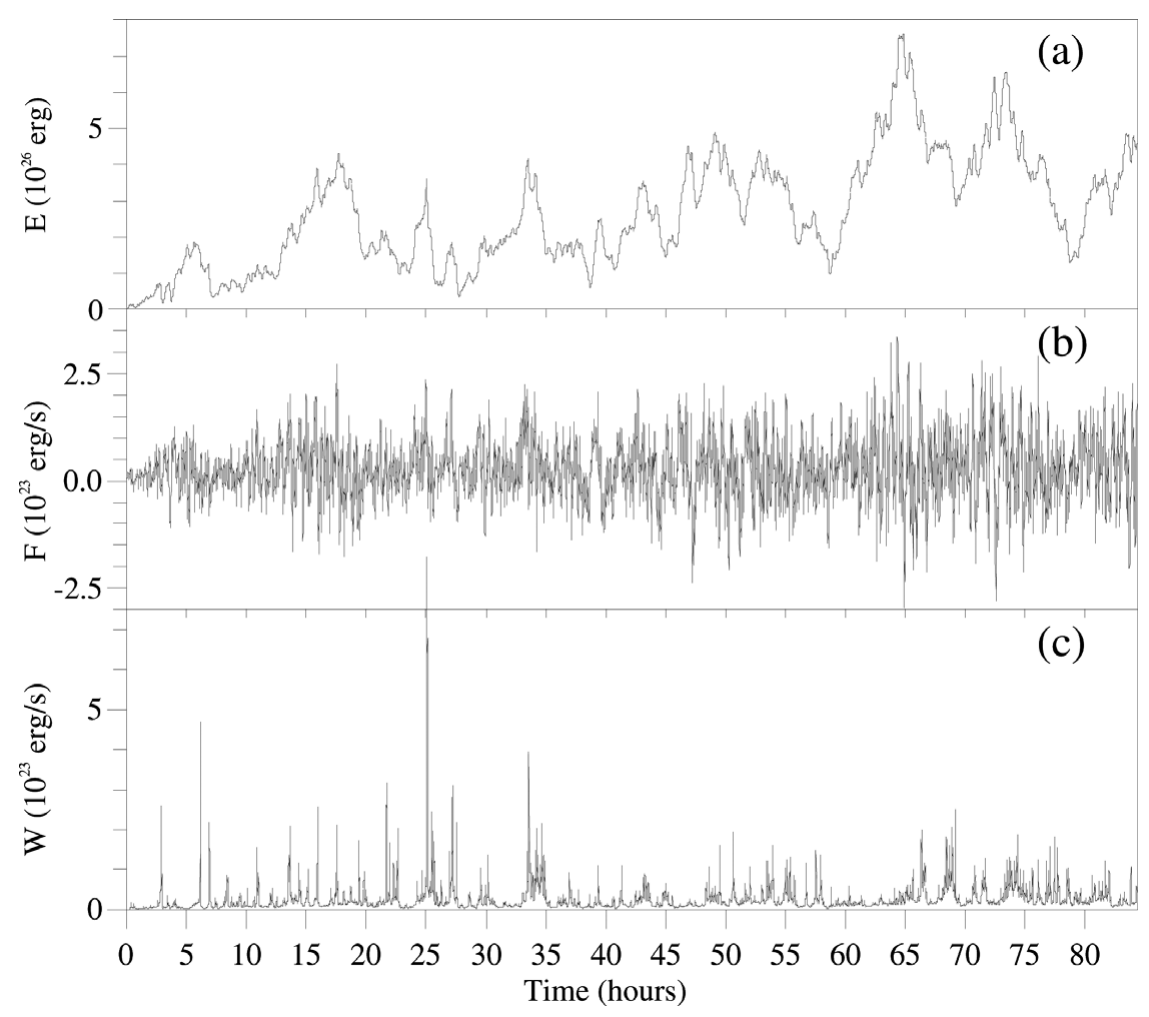

Figure 4. Total energy $E$ (upper panel), net incoming power $F$ (middle panel) and dissipated power $W$ (lower panel) as functions of time $t$.

\section{Conclusions}

We have examined and discussed some features of the energy input in a coronal loop due to photospheric motions at large transverse scales and of the subsequent generation of a turbulence towards smaller scales, where this energy can be dissipated. We focused on the energy injection range which is dominated by coherent dynamics. Perturbations are essentially formed by resonant eigenmodes at discrete frequency multiples of the fundamental Alfvén frequency, including the eigenmode at $\omega=0$ corresponding to DC perturbations. The latter is mainly responsible for the input energy flux, that essentially depends on the coupling between such an eigenmode and the external driver. The coherence properties of eigenmodes imply that at large scales nonlinear effects are much smaller than linear ones (weak nonlinearity). In this limit, the input flux is independent of nonlinear effects. The spectral flux is determined by interactions between AC perturbations at times $\sim T_{A}$ and the velocity perturbation of DC modes: then, both AC and DC mechanisms regulate the energy balance of the loop. Moreover, the weak nonlinearity allows for the formation of a pre-inertial range of the turbulence, where nonlinear interactions are influenced by coherence effects: a dominance of magnetic on kinetic energy and a reduced spectral energy flux. This theory has given scaling laws for the input energy flux $\left\langle\Phi_{\text {tot }}^{i n}\right\rangle_{t}$, the spectral flux $\left\langle\Phi_{\text {tot }}^{n l}\right\rangle_{t}$ from large to small scales, and the velocity perturbation level $\delta v$. The estimation for $\left\langle\Phi_{t o t}^{i n}\right\rangle_{t}$ is in agreement with the energy flux required to sustain the quiet-Sun corona against radiative losses (Withbroe 1988), while $\delta v$ is of the order of nonthermal velocities deduced from nonthermal broadening (e.g., 


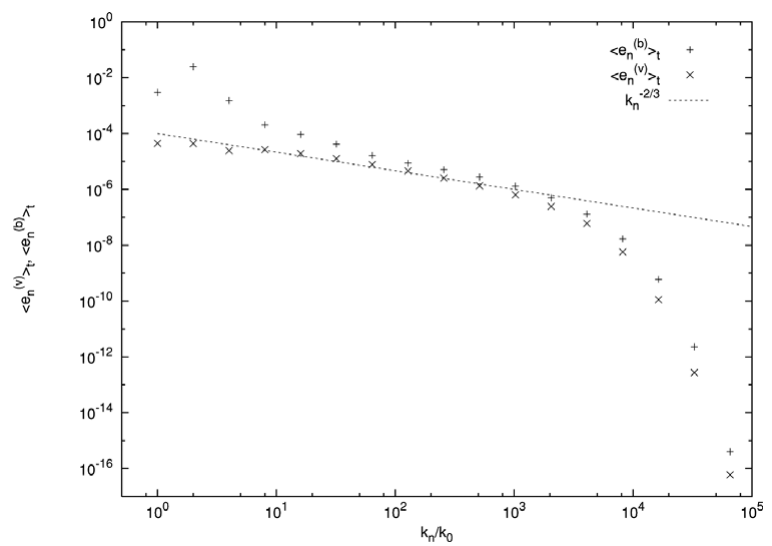

Figure 5. Spectra $\left\langle e_{n}^{(v)}\right\rangle_{t}$ of velocity perturbation ( " $\times$ " symbols) and $\left\langle e_{n}^{(b)}\right\rangle_{t}$ of magnetic field perturbation ("+" symbols) as functions of the transverse wavevector $k_{\perp n}$. The line corresponds to a Kolmogorov spectrum.

Warren et al. 1997). All the above properties have been checked on a numerical model (the hybrid shell model) finding a good agreement.

\section{References}

Boffetta, G., Carbone, V., Giuliani, P., Veltri, P., \& Vulpiani, A., 1999, Phys. Rev. Lett., 83, 4662

Buchlin, E. \& Velli, M., 2007, ApJ, 662, 701

Davila, J. M. 1987, ApJ, 317, 514

Dmitruk, P. \& Gomez, D. O., 1997, ApJ, 484, L83

Einaudi, G., Velli, M., Politano, H., \& Pouquet, A., 1996, ApJ, 457, L113

Giuliani, P. \& Carbone, V., 1998, Europhys. Lett., 43, 527

Heyvaerts, J. \& Priest, E. R., 1983, Astron. Astrophys., 117, 220

Ionson, J. A., 1982, ApJ, 254, 318

Milano, L., Gomez, D. O., \& Martens, P. C. H., 1997, ApJ, 490, 442

Nigro, G., Malara, F., Carbone, V., Veltri, P. 2004, Phys. Rev. Lett., 92, 194501, 1.

Nigro, G., Malara, F., \& Veltri, P. 2008, ApJ, 685, 606

Petkaki, P., Malara, F., \& Veltri P. 1998, ApJ, 500, 483

Strauss, H. 1976, Phys. Fluids, 19, 134

Sturrock, P. A. \& Uchida, Y. 1981, ApJ, 246, 331

Withbroe, G. L., 1988, ApJ, 325, 442

Warren, H. P., Mariska, J. T., Wilhelm, K., \& Lamaire, P. 1997, ApJ, 484, L91

\section{Discussion}

DAVILA: I wonder if the Reduced MHD solutions violate the initial assumptions used to derive the equations originally.

MALARA: Reduced MHD is based on the hypothesis that perpendicular gradients are made larger than parallel gradients. When a current sheet forms (at large scales) the associated gradient is quasi-perpendicular to B, so the above assumption is not violated. Moreover, perturbations responsible for the current sheet formation are included in these approaches as DC perturbations ( $\omega=0$ resonant eigenmodes). 
TsAP: Why do you think about the fact that the crossing Alfven time in the solar atmosphere is about $20 \mathrm{~s}$ ? Its value is significantly greater in the photosphere.

MALARA: I tried to estimate how much the first-order resonance frequency decreases when chromosphere is included. For this I used a simple exponential dependence of the Alfven speed on the altitude. I found that this frequency is decreased by a factor of 3 , while the input energy flux is only slightly increased. 